\title{
Simple pressure gauge for uranium hexafluoride
}

\author{
Patricia M. George, Mark N. Swanson, and J. L. Beauchamp \\ Arthur Amos Noyes Laboratory of Chemical Physics, California Institute of Technology, Pasadena, California 91125 \\ (Received 18 September 1978; accepted for publication 6 July 1979)
}

\begin{abstract}
A sensitive detector and pressure gauge for uranium hexafluoride in high-vacuum systems is described. Negative surface ionization of $\mathrm{UF}_{6}$ occurs on ribbon filaments operated at temperatures too low for electron emission to be significant. The ion current measured on a cylindrical collector surrounding the filament assembly varies regularly with $\mathrm{UF}_{6}$ pressure below $10^{-3}$ Torr. Different filament materials are considered, including rhenium, thoriated tungsten, and platinum. Rhenium is found to be the most satisfactory material for operation of diode emitters as a pressure gauge. Gauge constants (in A Torr ${ }^{-1}$ ) are derived from comparing negative surface ionization currents with the response of a capacitance manometer and are shown to be independent of temperature within a reasonable operating range. The effects of exposing the rhenium filament to various gases is considered, and it is shown that brief exposure to acetylene substantially improves the operating characteristics of the gauge.
\end{abstract}

\section{INTRODUCTION}

Recent experimental studies of the behavior of uranium hexafluoride in ionized gases in our laboratory ${ }^{1,2}$ led to the observation that ionization gauges of conventional design experience difficulties when exposed to this species. Although inverted Bayard-Alpert ionization gauges appear to respond properly to $U_{6}$, the thoriacoated iridium filaments commonly used in these gauges have short lifetimes even at relatively low pressures of $U_{6} \quad\left(10^{-6}\right.$ Torr $)$. Schulz-Phelps-type gauges, using rhenium wire filaments, behave erratically when exposed to $\mathrm{UF}_{6} \cdot{ }^{1}$ Deposits of thermally decomposed $\mathrm{UF}_{6}$ accumulate on heated surfaces of both gauges and further interfere with their proper operation.

The electron affinity of $U F_{6}$ is exceptionally high (5.2 $\mathrm{eV}^{2,3}$ ) and is comparable to the work functions of many metals. The resulting efficient negative surface ionization of $\mathrm{UF}_{6}$ on metal surfaces formed the basis of a $\mathrm{UF}_{6}{ }^{-}$ion beam source in our earlier experiments, ${ }^{2}$ and it was suggested that this phenomenon could be further exploited in the design of a detector for uranium hexafluoride. Subsequent results of Compton et $\mathrm{al} .{ }^{4}$ have shown that large amounts of $\mathrm{UF}_{6}{ }^{-}$are formed when fluorine gas is passed over heated uranium wire. The temperature dependence of $\mathrm{UF}_{6}{ }^{-}$, formed in this way, was found to be approximately the same as that formed by passing $\mathrm{UF}_{6}$ over a hot uranium wire. Recently, the formation of $\mathrm{UF}_{6}{ }^{-}$from $\mathrm{UF}_{6}$ on a carbon-coated platinum surface ${ }^{5}$ has been reported. This proceeds with an absolute efficiency of $\sim 99 \%$, the heat of adsorption of $\mathrm{UF}_{6}{ }^{-}$being $33.6 \pm 1.1 \mathrm{kcal} \mathrm{mol}^{-1}$.

This paper reports additional studies of the negative surface ionization of $\mathrm{UF}_{6}$ on various metals and the design, operation and calibration of a simple detector and pressure gauge for $\mathrm{UF}_{6}$.

\section{EXPERIMENTAL}

An all stainless-steel vacuum system pumped with a liquid nitrogen trapped 2 -in. diffusion pump was as- sembled. After bakeout at $200^{\circ} \mathrm{C}$ the base pressure was $5 \times 10^{-8}$ Torr. A stainless-steel sample reservoir containing $60 \mathrm{~g}$ of $\mathrm{UF}_{6}$ was attached to the vacuum system through a Granville Phillips variable leak valve. The sample reservoir was maintained at $0{ }^{\circ} \mathrm{C}$ in an ice bath to reduce the vapor pressure of $\mathrm{UF}_{6}$ and prevent distillation into other parts of the apparatus. Pressures below $10^{-4}$ Torr were monitored using a Bayard-Alpert ionization gauge (Veeco type $R G 75 \mathrm{~K}$ ). In the presence of $U F_{6}$, excessive currents were required to maintain regulated electron emission, leading to eventual failure of the filament. The gauge was replaced several times during the course of this investigation. At higher pressures (above $10^{-4}$ Torr) a capacitance manometer was employed (MKS Instruments, model 221).

A filament holder with cylindrical collector (Fig. 1) was assembled on a 2.75-in. flange and mounted in the vacuum system such that the filament could be viewed with an optical pyrometer through a small aperture in the cylindrical collector. Optical access to the assembly was provided by a quartz window. Rhenium, thoriated tungsten, and platinum ribbon filaments of length $1.3 \mathrm{~cm}$ and various widths were used. The collector was operated at ground and a variable negative bias was applied to the filament to extract negative ions. A negative bias of $300 \mathrm{~V}$ was maintained for calibration of the system as a pressure gauge.

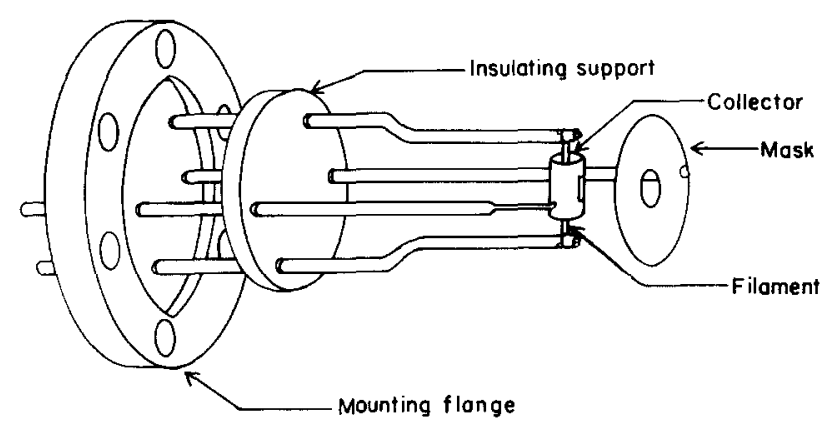

FIG. 1. Apparatus for investigation of $U_{6}$ negative surface ionization on filaments on various materials. 
For a given filament, the thermionic emission current was measured as a function of the apparent filament temperature $\left(T_{i}\right)$, determined with an optical pyrometer (Leeds and Northrup model 8622-C), and the true temperature $(T)$ was found from the equation ${ }^{6}$

$$
T^{-1}=T_{i}^{-1}+\left(\lambda_{i} / c_{2}\right) \ln \left[q \epsilon\left(\lambda_{i}\right)\right],
$$

where $\epsilon\left(\lambda_{i}\right)$ is the emissivity of the filament at wavelength $\lambda_{i}, 650 \mathrm{~nm}$, and temperature $T, c_{2}$ is the second radiation constant, and $q$ is the transmission of the quartz window.

The electronic work function of the filament $\phi$ was obtained by applying the Richardson-Dushman equation ${ }^{7}$

$$
J=A T^{2} \exp (\phi / k T),
$$

where $J$ is the saturation electron current density emitted from the surface at absolute temperature $T, A$ is a constant, and $k$ is the Boltzmann constant. Work functions can vary with both temperature and exposure to various gases (especially $\mathrm{UF}_{6}$ ). Optically determined work functions have the advantage that they can be measured at a fixed temperature. In the present study these were simply determined by varying the wavelength of the
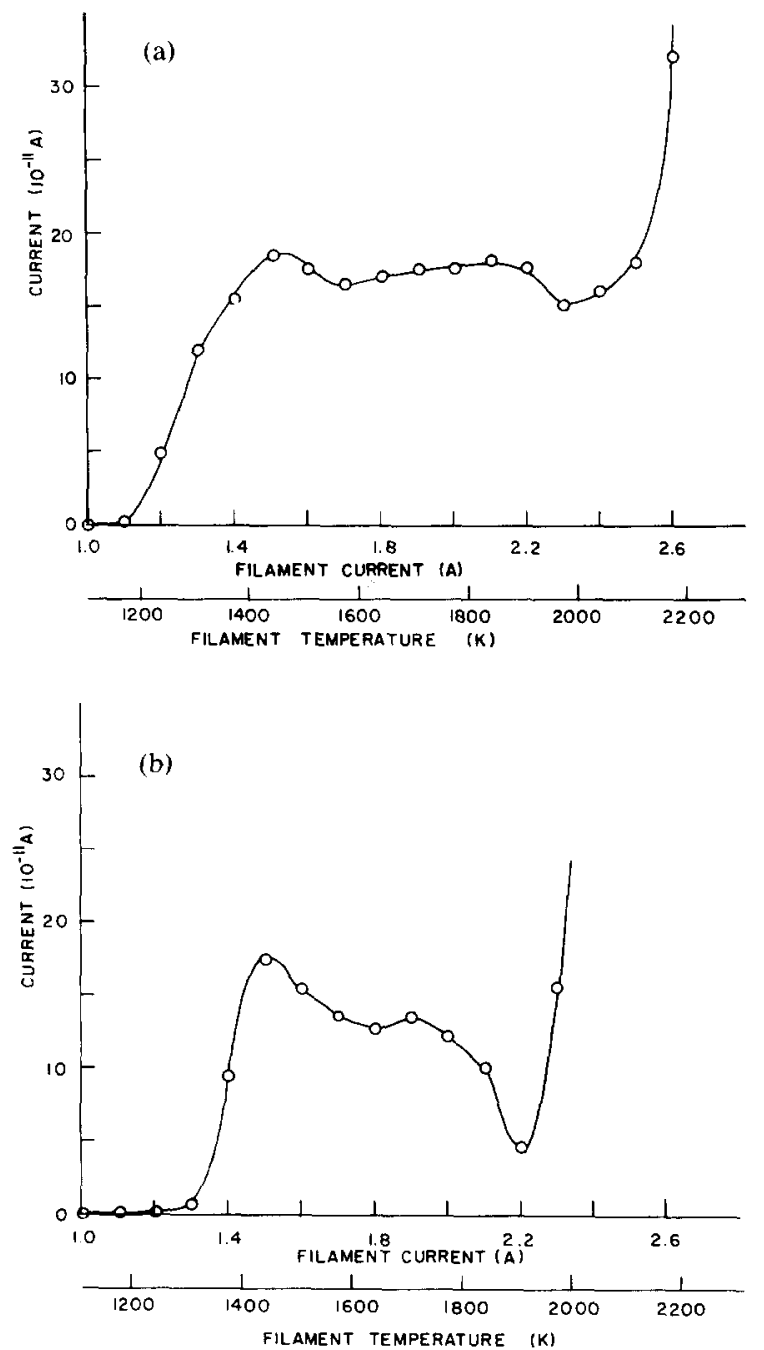

FIG. 2. (a) and (b). Representative data showing variation of negative collector current with rhenium flament current and temperature in the presence of $2 \times 10^{-5}$ Torr of $\mathrm{UF}_{6}$.

\begin{tabular}{|c|c|c|c|c|c|}
\hline \multirow[b]{2}{*}{ Filament } & \multirow[b]{2}{*}{$\epsilon_{0.65}{ }^{\mathrm{h}}$} & \multicolumn{3}{|c|}{ Electronic work function $(\mathrm{eV})$} & \multirow[b]{2}{*}{ MP K } \\
\hline & & Thermionic & $\begin{array}{l}\text { Photo- } \\
\text { electric }\end{array}$ & $\begin{array}{l}\text { Liter- } \\
\text { ature }^{\mathrm{d}}\end{array}$ & \\
\hline $\operatorname{Re}$ & 0.42 & 4.7 & 4.5 & 4.96 & 3453 \\
\hline $\mathrm{W}(\mathrm{Th})^{\mathrm{a}}$ & 0.43 & 4.4 & $\sim 4.5$ & 4.55 & 3683 \\
\hline $\mathrm{Pt}_{\mathrm{t}}$ & 0.33 & 3.2 & - & 5.65 & 2042 \\
\hline
\end{tabular}

TABLE I. Average electronic work functions of filament materials.

"Thorium depleted by strong heating.

"W. H. Kohl, Materials and Technigues for Electron Tuhes (Reinhold, New York, 1960).

"Handbook of Chemistry and Physics. 53rd ed. (Chemical Rubber Company, Cleveland, 1972).

" H. B. Michaelson, J. Appl. Phys. 48, 4729 (1977).

incident light and observing the threshold for photoelectron emission. ${ }^{8}$ The filament was illuminated with a $150-\mathrm{W}$ xenon light source, dispersed by a monochromator (Bausch and Lomb, $0.25 \mathrm{~m}$ ). A mask allowed the light to strike the filament but not the collector.

Pressure-gauge calibrations were carried out using a $0.0012 \times 0.030$ in. rhenium ribbon. Gauge constants were determined for treated and untreated rhenium filaments. Treatment involved passing acetylene at $5 \times 10^{-5}$ Torr over the heated filament $(\sim 1400 \mathrm{~K})$ for $\sim 10 \mathrm{~min}$ before allowing $\mathrm{UF}_{6}$ into the system. This forms a surface carbide on the filament and typically enhances formation of $\mathrm{UF}_{6}-$ by $\sim 10^{3}$ over that of untreated filaments. The rhenium filaments were degassed and cleaned by heating to $\sim 2300 \mathrm{~K}$ for several minutes before use.

Rhenium filaments subjected to different operating conditions were examined with a scanning electron microscope to identify changes in surface characteristics.

\section{RESULTS}

\section{A. Rhenium}

In an earlier study in this laboratory, ${ }^{2}$ it was observed that $\mathrm{UF}_{6}{ }^{-}$was formed on a rhenium filament at filament temperatures too low for electron emission to be significant. Detection and characterization of $\mathrm{UF}_{6}{ }^{-}$was accomplished by ion cyclotron resonance spectroscopy. The mass spectrum showed no other major negative ions produced on the filament. Small amounts of rhenium hexafluoride were, however, detected in the positive-ion mass spectrum. At a $\mathrm{UF}_{6}$ pressure (measured on an ionization gauge) of $1 \times 10^{-6}$ Torr, the onset for $\mathrm{UF}_{6}{ }^{-}$emission occurred at a filament temperature of about $1100 \mathrm{~K}$, while electron emission did not occur until $\sim 2000 \mathrm{~K}$. This behavior has been confirmed in this study; the variation of negative-ion current with filament temperature for a $0.0012 \times 0.030$ in. rhenium ribbon is shown in Fig. 2 . $\mathrm{UF}_{6}{ }^{-}$is initially observed in the temperature range $1000-1200 \mathrm{~K}$. The negative-ion current steadily increases with temperature until it reaches a maximum. Thereafter, it either remains at a maximum, Fig. 2(a), or decreases, Fig. 2(b), until electron emission is observed at higher temperatures. Location of this maximum and subsequent behavior of the $\mathrm{UF}_{6}{ }^{-}$current depends on individual filaments and the condition of that filament, but in all 


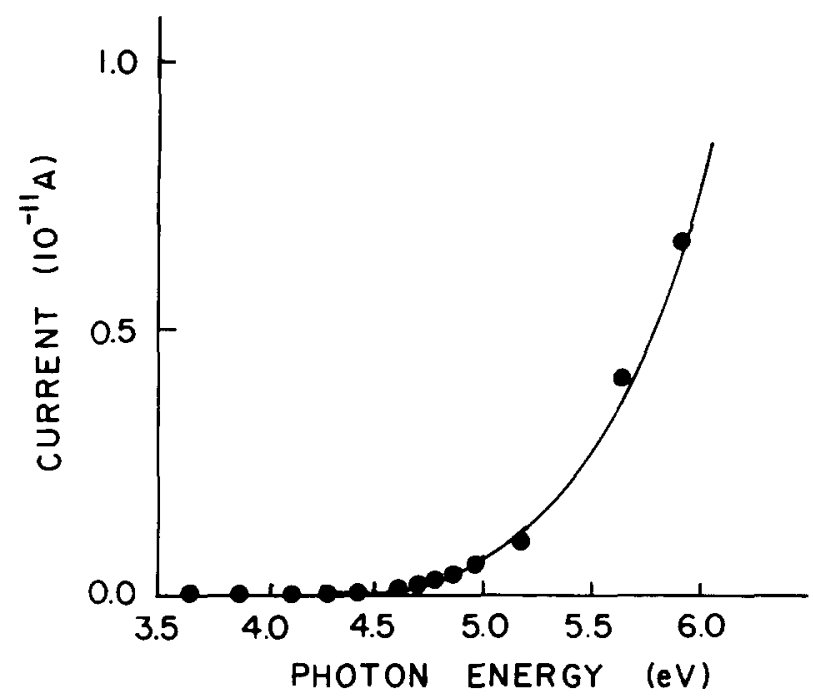

FIG. 3. Variation of electron current with frequency of light impinging on rhenium surface. Threshold extrapolation of work function yields a value of $4.5 \mathrm{eV}$.

cases maximum emission is readily located. At this point the negative ion current is flux dependent and varies linearly with pressure (see below). Operating range for the detector and the pressure gauge is in the vicinity of this maximum $(1200-1600 \mathrm{~K})$.

With a new rhenium filament mounted, analysis of the electron emission as a function of temperature yielded an average work function of $4.7 \mathrm{eV}$ (a summary of work functions obtained is given in Table $\mathrm{I}$ ). The filament was then exposed briefly to $U F_{6}$ and $U F_{6}{ }^{-}$emission was observed with an apparent low negative surface ionization efficiency of $1.3 \times 10^{-7}$. Upon removal of $U F_{6}$ vapor, thermionic emission measurements indicated that the work function had increased to $5.5 \mathrm{eV}$. This increase in work function was also demonstrated using photoelectric techniques. Figure 3 shows the observed photocurrent before contact with $\mathrm{UF}_{6}$, threshold extrapolation of which yields a value of $4.45 \mathrm{eV}$ for the work function. Irradiation of the rhenium ribbon with $200 \mathrm{~nm}(6.2 \mathrm{eV})$ light yielded a photoelectric current of $1 \times 10^{-11} \mathrm{~A}$. Exposure to $\mathrm{UF}_{6}$ immediately quenched the current, which could only be restored by pumping away the $U F_{6}$ and strongly heating the filament. This suggests that the work function at ambient temperature (295 K) increases by over $1.6 \mathrm{eV}$ upon exposure to $\mathrm{UF}_{6}$, since the photoelectric threshold is now greater than $6.2 \mathrm{eV}$.

Electron micrographs show that initially the rhenium surface is devoid of any distinctive features. Deposits which appear to be $\mathrm{UF}_{4}$ are formed after exposure to $\mathrm{UF}_{6}$ at elevated temperatures. In addition to the deposits, surface striations are clearly visible. The filament operates in this condition but with low efficiency.

\section{B. Thoriated tungsten}

Thoriated tungsten was chosen since it is extensively used as filament material. However, prior heating to $2850 \mathrm{~K}$ of the $0.002 \times 0.040$ in. filament caused substantial depletion of thorium, so that the properties of the fila- ment were essentially those of tungsten. ${ }^{9}$ At a $U F_{6}$ pressure of $4 \times 10^{-5}$ Torr, the variation of negative-ion current with filament temperature produced a curve similar to that for rhenium, with a maximum $U F_{6}$ current of $5.6 \times 10^{-8} \mathrm{~A}$ at $1100 \mathrm{~K}$. The ionization efficiency for these filaments is $1 \times 10^{-3}$, but prolonged exposure to $U F_{f i}$ lead to erosion and failure of the filament.

The thermionic work function of the clean filament was found to be $4.4 \mathrm{eV}$, while the photoelectric work function yielded a value of $4.5 \mathrm{eV}$. As was the case for rhenium, $\mathrm{UF}_{6}$ caused an increase in the work function of the metal. Allowing $U_{F}$ into the system eliminated the photoelectron current due to a $200-\mathrm{nm}$ light impinging on the filament surface, and the work function remained high (5.3 $\mathrm{eV}$ in one measurement) for some time even after removal of $U F_{6}$ vapor.

\section{Platinum}

The $0.005 \times 0.029$ in. platinum filament was heated to $1250 \mathrm{~K}$ for $\sim 2 \mathrm{~h}$ in order for the filament to outgas, after which the thermionic work function was found to be $3.2 \mathrm{eV}$. Consistent results were difficult to obtain, however, probably due to contamination by hydrocarbons, although this platinum is the material used for filaments in several applications. Of the untreated filaments investigated, platinum proved the most efficient in producing $\mathrm{UF}_{6}$. Again, at a $\mathrm{UF}_{6}$ pressure of $4 \times 10^{-5}$ Torr a curve similar to that in Fig. 2(b) was obtained; the maximum $\mathrm{UF}_{6}$ current being $2.2 \times 10^{-5} \mathrm{~A}$. The ionization efficiency is $\sim 0.5$, much greater than that of the metals considered above. In spite of this high surface ionization efficiency, platinum was regarded as unsuitable as a filament material due to the repeated failure of the ribbon in a short period of time. Failure is attributed to surface reactions which erode the filament and lead to the development of hot spots. Another major drawback to the use of platinum is that it begins to volatize at $\sim 1570 \mathrm{~K}$ and melts at $2042 \mathrm{~K}$.

\section{Calibration of pressure gauge using rhenium filament}

On the basis of the above results, rhenium was chosen for use in the pressure gauge. The maximum $U F_{6}{ }^{\prime \prime}$ current produced in this filament varies regularly with $\mathrm{UF}_{6}$ pressure up to $10^{-3}$ Torr. The extraction bias is fixed at $300 \mathrm{~V}$, which is above the value where the diode emitter is spacecharge limited. ${ }^{10}$

Calibration of the pressure gauge was accomplished by recording $\mathrm{UF}_{6}{ }^{-}$current versus $\mathrm{UF}_{6}$ pressure, measured by the capacitance manometer. Figure 4 shows the results of these measurements for the clean rhenium filament at temperatures varying from $1403-1583 \mathrm{~K}$. The linearity is excellent in the range $10^{-4}-10^{-3}$ Torr, where the capacitance manometer provides reliable readings. Although not directly substantiated by our experiments, it is expected that the linearity extends to much lower pressures where the $\mathrm{UF}_{6}$ gauge still responds. Consistent with this expectation is the observed extrapolation of the 
data in Fig. 4 to zero pressure on both axes. The gauge constant (in A Torr ${ }^{-1}$ is given by the slope of the line and it can be seen that this does not vary within the operating temperature range. Similar results were obtained for filaments treated with acetylene. Pressure gauge constants for treated and untreated rhenium filaments are $1.7 \pm 0.1$ $\times 10^{-7} \mathrm{~A} \mathrm{Torr}^{-1}$ and $1.1 \pm 0.3 \times 10^{-4} \mathrm{~A} \mathrm{Torr}^{-1}$, where the error limits are the standard deviations from the respective means. Acetylene treatment does increase the ion current by a factor of $\sim 1000$. However, it has the disadvantage that the characteristic $\mathrm{UF}_{6}{ }^{-}$emission in Fig. 2 often disappears after a few hours of $U_{6}$ exposure and electron emission alone is observable at higher temperatures. Repeated use of acetylene, on the other hand, can revive the filament for short periods.

\section{DISCUSSION}

This study demonstrates that the simple device of Fig. 1 is very sensitive to $U_{6}$, producing currents which vary rapidly and in a reproducible fashion with $\mathrm{UF}_{6}$ pressures below $10^{-3}$ Torr.

The effect of $\mathrm{UF}_{6}$ on the rhenium filaments has not been fully characterized. However, it appears that $U F_{6}$ is chemisorbed on the rhenium in two forms, the first being $\mathrm{UF}_{6}{ }^{-}$and the second being decomposed $\mathrm{UF}_{6}$. If one assumes that the heat of adsorption for $\mathrm{UF}_{6}{ }^{-}$on rhenium is similar to that on carburized platinum ${ }^{11}$ given by Dittner and Dat $z^{5}$ it can be calculated from simple chemisorption theory ${ }^{12}$ that the radius of the adsorbed ion is approximately $3.5 \AA$. Assuming point charges, a $\mathrm{Re}-\mathrm{UF}_{6}$ dipole moment of $23.5 \mathrm{D}$ is calculated, from which the contact potential at complete coverage is estimated to be $4.4 \mathrm{eV}$. This suggests $-20 \%$ coverage for the observed $0.8 \mathrm{eV}$ increase in work function. Similar calculations for decomposed $\mathrm{UF}_{6}$, in the form of $\mathrm{UF}_{n}$ and $\mathrm{F}$ (the fluorine being chemisorbed while the $\mathrm{UF}_{n}$ is physisorbed) yields a contact potential of $0.5 \mathrm{eV}$ at $20 \%$ coverage. Some of the $\mathrm{UF}_{6}$ leaves the surface as negative ions, but it would appear that the work function increase is due to both chemisorbed $\mathrm{UF}_{6}$ and chemisorbed fluorine, the physisorbed material being removed when the filament is heated. It has been suggested previously that a layer of halogen atoms on a metal surface produces a dielectric layer that effectively increases work function of the metal. ${ }^{\text {? }}$

Ionization efficiencies obtained in these experiments are much lower than those calculated from the SahaLangmuir equation. This is in agreement with the findings of Dittner and Datz ${ }^{5}$ for the formation of $\mathrm{UF}_{6}{ }^{-}$on clean platinum. The temperature dependence of the $\mathrm{UF}_{6}{ }^{-}$current exhibited in Fig. 2 can be understood in qualitative terms by the covering effect of $U_{6} \cdot{ }^{7}$ When the rhenium is exposed to $\mathrm{UF}_{6}$ the work function increases due to development of an adsorption layer on the surface as noted above. The temperature dependence of the $\mathrm{UF}_{6}$ yield at low temperature is consistent with this increase in work function, provided that it exceeds the electron affinity of $\mathrm{UF}_{6}(5.2 \mathrm{eV}){ }^{2}$ At higher temperatures (1600-

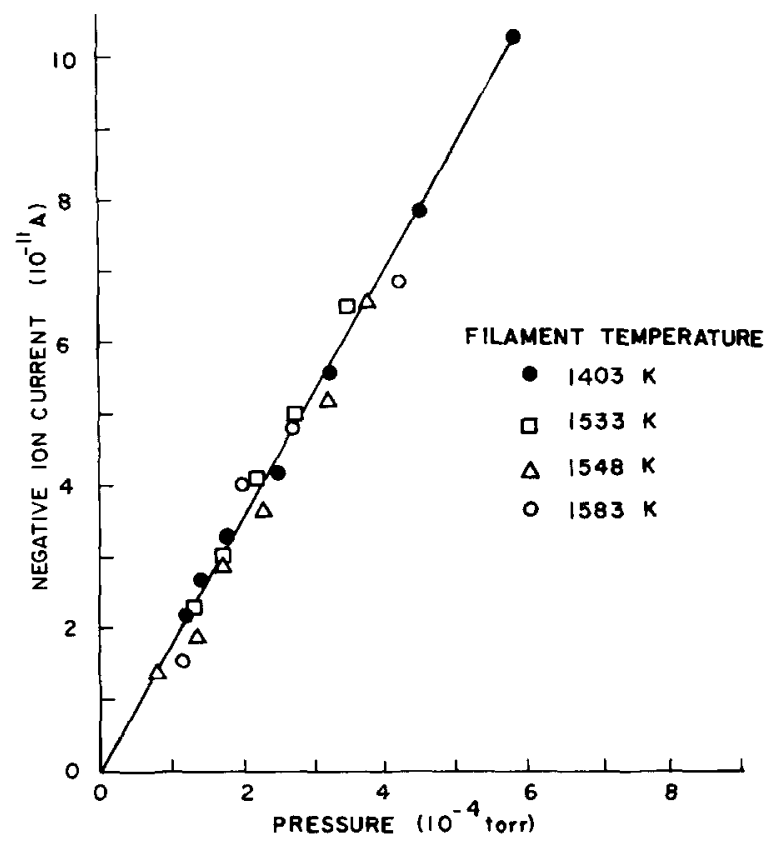

FIG. 4. Variation of $U F_{6}$ current with pressure of $U F_{6}$ for a clean rhenium filament.

$2000 \mathrm{~K}$ ) decomposition of $\mathrm{UF}_{6}$ on the filament surface competes with $\mathrm{UF}_{6}{ }^{-}$formation. Decomposition may react with the rhenium to further modify the work function. Hence the $\mathrm{UF}_{6}{ }^{-}$emission current may decrease or remain constant depending on the condition of the filament.

Conventional ionization gauges exposed to $\mathrm{UF}_{6}$ are afflicted by an increase in work function which necessitates that they be operated at higher temperatures. One possible way of increasing the lifetime of an ionization gauge which is regularly exposed to $\mathrm{UF}_{6}$ would be to replace the commonly used thoria-coated iridium filament with rhenium ribbon, which has a higher melting point and which appears to be fairly well behaved in the presence of $\mathrm{UF}_{6}$. Schulz-Phelps gauges are unsuitable for $\mathrm{UF}_{6}$ pressure measurements because both negative ions and electrons are registered as the emission current. Since emission currents in such gauges are typically $10^{-5}-10^{-6} \mathrm{~A}$, ion emission can be a substantial fraction of the total. A problem which occurs with all filaments, however, is that the products of thermal decomposition of $\mathrm{UF}_{6}$ can react with and erode the surface. In the case of rhenium, rhenium hexafluoride was detected by its positive-ion electron-impact mass spectrum.

As a $\mathrm{UF}_{6}$ detector and pressure gauge, the device can be used for pressures below $10^{-3}$ Torr. Disadvantages of the device arise if a high pressure of $\mathrm{UF}_{6}$ is sustained for too long a period of time. These are manifested by filament poisoning which may or may not be reversible with acetylene treatment. However, the simplicity of design and use of the gauge seems to adequately compensate for such difficulties which can be minimized by operating at the lowest possible temperature. Initially, it is advisable to calibrate the negative-ion current against an absolute gauge for the particular filament used. On the basis of this study, rhenium is the filament material suggested. There is no need to read filament temperatures since, 
after exposure to $\mathrm{UF}_{6}$, the maximum emission of $\mathrm{UF}_{6}$ at low filament currents can be located. Operation at this maximum guarantees that the gauge response will not change markedly with filament current.

\section{ACKNOWLEDGMENT}

This work was supported in part by the United States Department of Energy under Grant No. E(04-3)767-8.

' J. L. Beauchamp, J. Chem. Phys. 64, 718 (1976).

2 J. L. Beauchamp, J. Chem. Phys. 64, 929 (1976).

${ }^{3}$ R. N. Compton, J. Chem. Phys. 66, 4478 (1977)

${ }^{4}$ R. N. Compton, P. W. Reinhardt, and W. R. Garrett, J. Chem. Phys. 66, 4712 (1977).

${ }^{5}$ P. F. Dittner and S. Datz, J. Chem. Phys. 68, 2451 (1978)

${ }^{6}$ C. M. Herzfeld, Temperature, Its Measurement and Control in
Science and Industry, Vol. 3 (Reinhold, New York, 1962), Pt. 1, p. 539 .

M. Kaminsky, Atomic and Ionic Impact Phenomena on Metal Surfaces (Springer-Verlag, Berlin, 1965), p. 136

${ }^{*}$ This method does not lead to a precise value for the work function. It does, however, facilitate investigation of changes in work functions at fixed temperatures (see Ref. 7.pp. 20-24 for a complete discussion).

" A. Persky, E. F. Green, and A. Kuppermann, J. Chem. Phys. 49, 2347 (1968).

1" R. G. Wilson and G. R. Brewer, Ion Beams with Applications to Ion Implantation (Wiley, New York, 1973), p. 91.

11 The heat of adsorption depends only on the work function of the surface, the $\mathrm{EA}\left(\mathrm{UF}_{6}\right)$, and the interaction energy between the ion and its electrical image. Since the last two terms are independent of the metal surface and the work function of $\operatorname{Re}(4.7 \mathrm{eV})$ is similar to the carbon-coated $\mathrm{Pt}$ surface (4.4 eV) it is felt this assumption is justified.

${ }^{12}$ For a complete discussion of simple chemisorption theory see. for example, (a) A. Clark, The Theory of Adsorption and Catalysis (Academic, New York, 1970), p. 169: or (b) Ref. 7, p. 52 